\title{
Research Article \\ Effects of Bisphosphonates on Osseointegration of Dental Implants in Rabbit Model
}

\author{
Ru Qing Yu, Jing Yi Wang, Nian Jing Rao, Lei Huo, and Li Wu Zheng \\ Discipline of Oral \& Maxillofacial Surgery, Faculty of Dentistry, The University of Hong Kong, Hong Kong SAR, China \\ Correspondence should be addressed to Li Wu Zheng; lwzheng@hku.hk
}

Received 23 October 2020; Accepted 29 January 2021; Published 12 February 2021

Academic Editor: Maciej Gagat

Copyright (C) $2021 \mathrm{Ru}$ Qing Yu et al. This is an open access article distributed under the Creative Commons Attribution License, which permits unrestricted use, distribution, and reproduction in any medium, provided the original work is properly cited.

\begin{abstract}
This study is to investigate the effect of bisphosphonates on the osseointegration of dental implants in a rabbit model. Twenty female New Zealand White rabbits were equally assigned into control and experiment groups which received saline or zoledronic acid treatment 4 weeks prior to surgery. Titanium dental implant was placed on the calvarial bone. Zoledronic acid or saline treatment continued after surgery for 4 weeks (short-term subgroup) or 8 weeks (long-term subgroup) until sacrifice. Three different fluorochrome labeling solutions were administrated for assessing bone growth rates. Samples of the calvarial bone and mandible were subjected to microcomputed tomography (micro-CT), confocal microscope, and histology analysis. Zoledronic acid treatment significantly reduced bone growth rates in the calvarial bone, but had no significant influence in bone mineral density and trabecular microarchitecture. Significantly lower bone-to-implant contact ratios were found in zoledronic acid-treated animals compared to controls at week 4 but not at week 8. Oncologic dose zoledronic acid suppresses the bone growth rates of the calvarial bone; ZA may have an adverse effect on osseointegration of dental implant in short term, but this effect tends to diminish in long term.
\end{abstract}

\section{Introduction}

Bisphosphonates work mainly on osteoclasts to reduce bone turnover rates and increase bone mineral density [1], mainly through their inhibitory effect on formation, differentiation, and activity of osteoblasts [2-7]. Bisphosphonates have been used to treat metabolic bone diseases, such as osteoporosis, Paget's disease, multiple myeloma, and bone metastasis [8]. Despite their great clinical benefits, a serious adverse event known as medication-related osteonecrosis of the jaws (MRONJ) has been reported.

Osseointegration is the principal success criteria of a dental implant [9], the concept of which was first discovered by Brånemark in 1969, demonstrating that it is a direct attachment between bone and the dental implant surface, with the competence of bearing the functional load [10]. Theoretically, the osseointegration of dental implants may benefit from bisphosphonates by reducing osteoclastic bone resorption and the subsequently diminishing bone remodeling; however, there is relatively limited data regarding the possible effects of bisphosphonates on relevant aspects of implant therapy, such as implant osseointegration, failure rate, and MRONJ development [11]. So far, there is no consensus concerning the placement of dental implants in patients receiving bisphosphonates. Most studies showed that low-dose oral $\mathrm{BP}$ for osteoporosis management does not compromise implant therapy, while there is almost no relevant information available on the possible effect on implant therapy of high-dose BPs [11].

The present study is aimed at evaluating the osseointegration of dental implants on a rabbit model treated with high-dose intravenous bisphosphonates in the long term and short term.

\section{Materials and Methods}

2.1. Study Design and Treatment. 22- to 26-week-old female New Zealand White rabbits ( $3.2 \mathrm{~kg}$ to $4.2 \mathrm{~kg}$ ) were randomly allocated into control and experiment groups, and each group was further divided into long-term and short-term subgroups with 5 rabbits in each subgroup (Table 1). Each rabbit was given zoledronic acid (experiment group, 
TABLe 1: Group allocation and treatment.

\begin{tabular}{lccc}
\hline Groups & No. & Treatments & Sacrifice \\
\hline \multirow{2}{*}{ Control } & 5 & Veh+implant insertion & Week 4 \\
& 5 & Veh+implant insertion & Week 8 \\
Experiment & 5 & ZA+implant insertion & Week 4 \\
& 5 & ZA+implant insertion & Week 8 \\
\hline
\end{tabular}

Veh: vehicle saline; ZA: zoledronate acid.

$0.1 \mathrm{mg} / \mathrm{kg}$, s/c) or vehicle saline (control group, $0.1 \mathrm{mg} / \mathrm{kg}$, $\mathrm{s} / \mathrm{c}$ ) three times per week for four weeks prior to surgery (animals underwent dental implant placement and tooth extraction. The tooth extraction was done for other investigation and will be presented in another article). The injection continued after the surgery until sacrifice. Animals in the short-term subgroups were sacrificed 4 weeks after surgery, and those in the long-term subgroups were sacrificed 8 weeks after surgery (Figure 1). In total, animals received 8 weeks and 12 weeks of saline or zoledronic acid in the short-term subgroups and long-term subgroups, respectively.

2.2. Dental Implant Insertion. All the implant placement was conducted under general anaesthesia. Heart rate, respiration rate, $\mathrm{SpO}_{2}$, and body temperature were recorded throughout the entire procedure.

The rabbit was placed in a prone position and shaved on the top of the head. The surgical site was disinfected, and a straight incision on the sagittal crest was performed. The calvaria was exposed after the subperiosteal dissection. Under continuous irrigation with sterile physiological $0.9 \%$ saline solution, a $3.5 \mathrm{~mm}$ craniotomy was performed with a rotating trephine burr and a round bur leaving dura mater intact, which was detached step by step from the inner table using first a small applicator and then a Freer periosteal elevator. A cylinder titanium implant (Straumann ${ }^{\circledR}$ SLA, $6 \mathrm{~mm}$, Basel, Switzerland) with a diameter of $4.1 \mathrm{~mm}$ was inserted, leaving $2 \mathrm{~mm}$ of the implant intracranially. The extracranial part of the implant was covered with a resorbable membrane (Geistlich Bio-Gide ${ }^{\circledR}, 25 \mathrm{~mm}$ $\times 25 \mathrm{~mm}$, Wolhusen, Switzerland). The scalp was closed after the epicranial aponeurosis was repositioned and closed over the membrane with single interrupted sutures $\left(\right.$ Ethilon $\left.{ }^{\circledR}\right)$. The sutures were removed after 2 weeks. Postoperative care including pain control and food care were given under guidance. Figure 2 briefly introduced the steps of the procedure.

2.3. Fluorochrome Labeling. Three different fluorochrome labeling solutions were prepared and injected in sequence subcutaneously $[12,13]$ : calcein green $(10 \mathrm{mg} / \mathrm{kg}$, SigmaC0875), alizarin complexone ( $30 \mathrm{mg} / \mathrm{kg}$, Sigma-A3882), and oxytetracycline (20 mg/kg, Sigma-O5875) at week 1, week 2, and week 3 after dental implant placement, respectively, in the short-term subgroups. For long-term subgroups which were sacrificed at week 8 , these fluorochrome labeling solu-

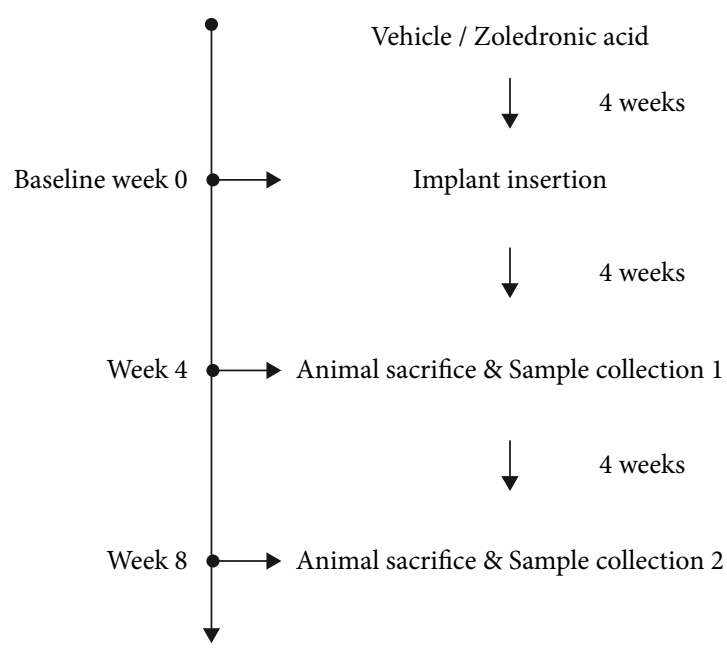

Figure 1: Timeline of the study. The baseline is set at the implant insertion day after 4 weeks of vehicle or ZA treatment. Sacrifice time points include 4 weeks and 8 weeks after implant insertion.

tions were injected at week 2 , week 4 , and week 6 , respectively.

2.4. Sacrifice and Sample Collection. All animals were euthanized by intravenous injection of ketamine through the ear vein. Calvaria containing the dental implant was resected (with a margin of which was no less than $10 \mathrm{~mm}$ away from the implant) and was preserved in $10 \%$ neutral buffered formalin solution for further examinations.

2.5. Microcomputed Tomography (Micro-CT) Examinations. To evaluate the bone volume, bone mineral density (BMD), and microarchitecture, all the samples were subjected to a microcomputed tomography (micro-CT) scanning (Skyscan1076; Bruker, Kontich, Belgium) at $88 \mathrm{kV}$ and $100 \mu \mathrm{A}$ intensity with a resolution of $17.3 \mu \mathrm{m}$ pixel. The reconstruction data were quantitatively analyzed with the CT analyzer software (version 1.9; Skyscan, Kontich, Belgium).

The region of interest (ROI) was selected as the circular bone tissue around the implant from the surface of the dental implant to the distance of $1.5 \mathrm{~mm}$ away from the implant centered on the midline of the trabecular area on the horizontal level (Figure 3). Data of BMD, BV (bone volume), $\mathrm{TV}$ (tissue volume), BV/TV (bone volume fraction, BV/TV = bone volume/tissue volume $[13,14])$, Tb.Th (trabecular thickness, 3D measures of the average thickness of the cancellous bone structure [14-15]), Tb.N (trabecular number, the number of trabecular plates per unit length [14-16][1416][14-16][14-16][13-15]), and Tb.Sp (trabecular separation, average diameter of the marrow cavities from ROI) were extracted for statistical analysis.

After micro-CT analysis, the undecalcified specimens were embedded in Technovit ${ }^{\circledR} 9100$ PMMA and later prepared to ground sections for fluorochrome labeling analysis and histological examinations with a final thickness of approximately $40-50 \mu \mathrm{m}$. Each section was made in the same 


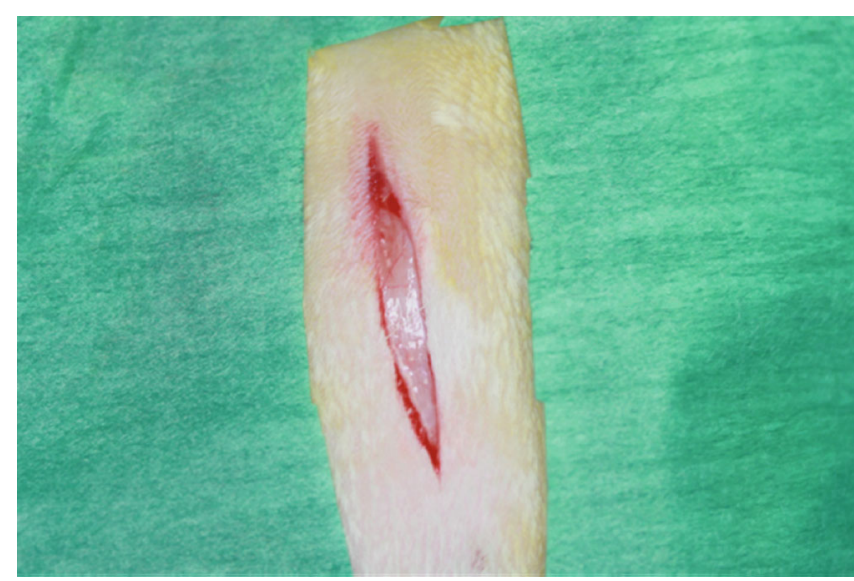

(a)

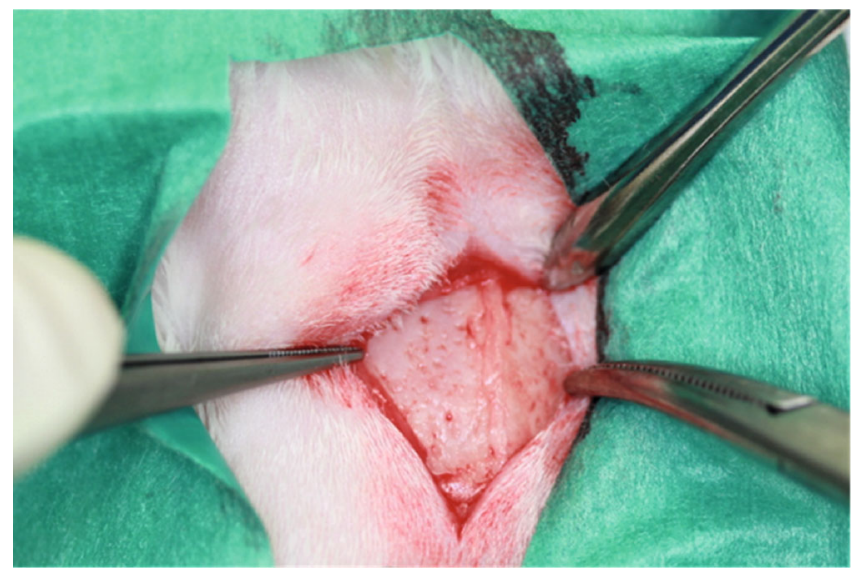

(c)

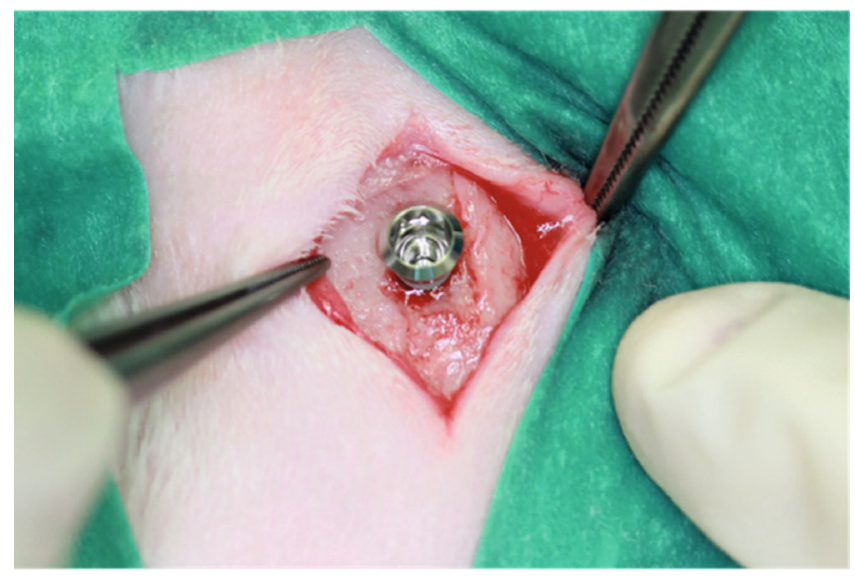

(e)

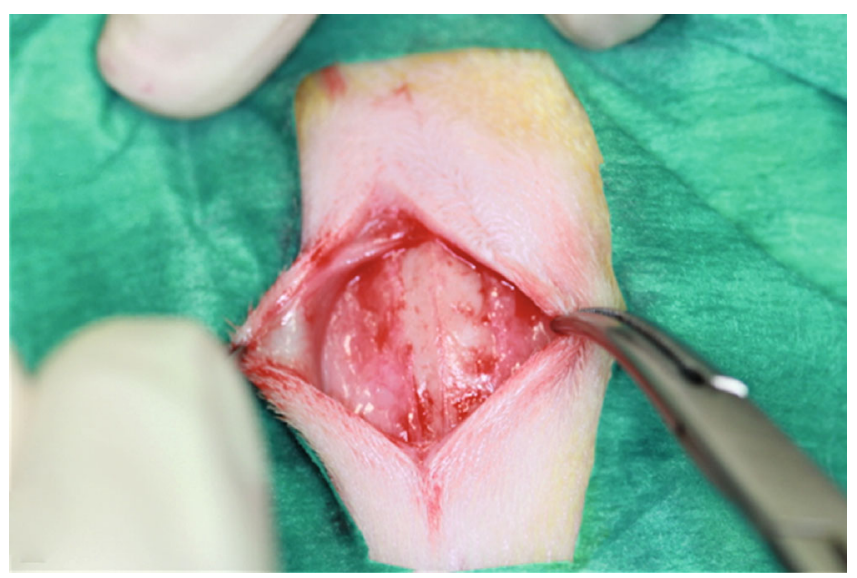

(b)

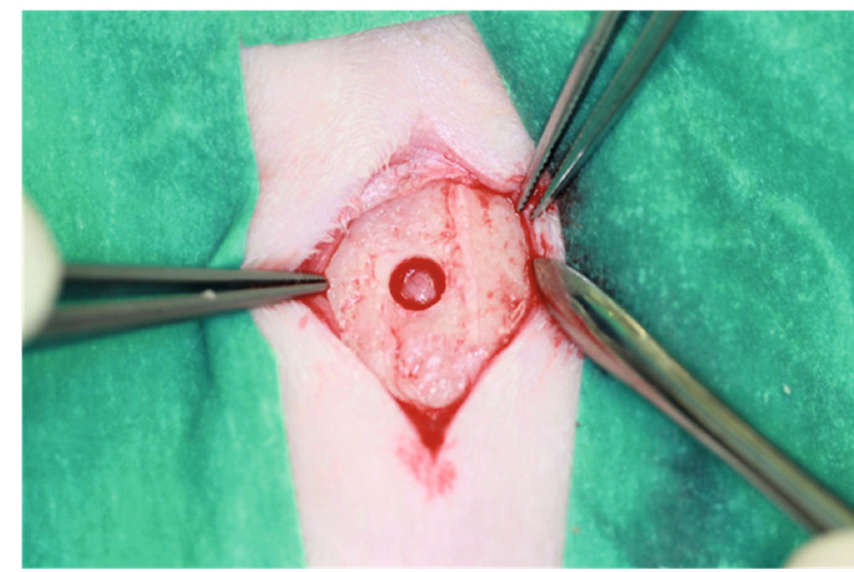

(d)

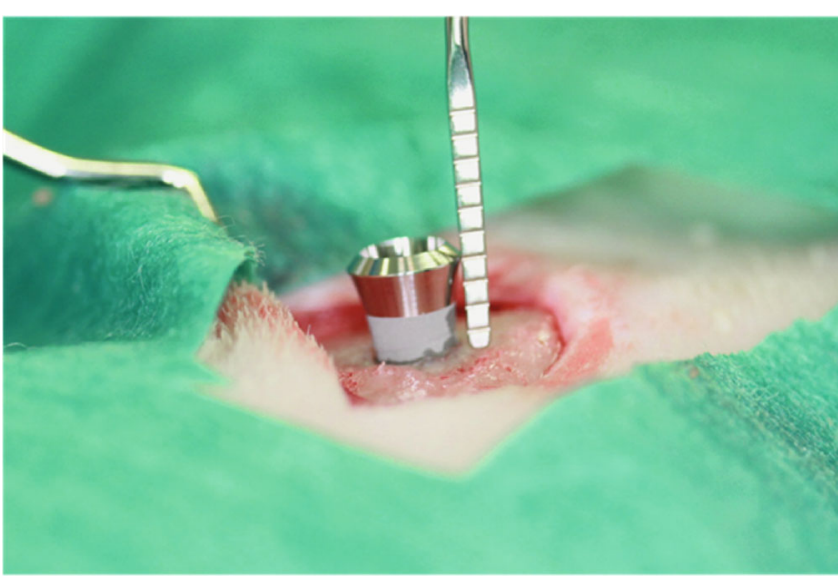

(f)

Figure 2: Continued. 


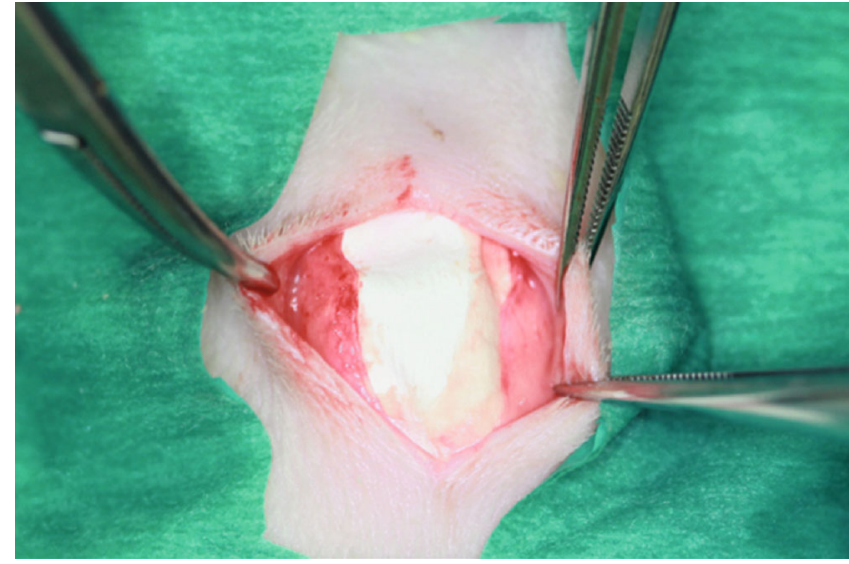

$(\mathrm{g})$

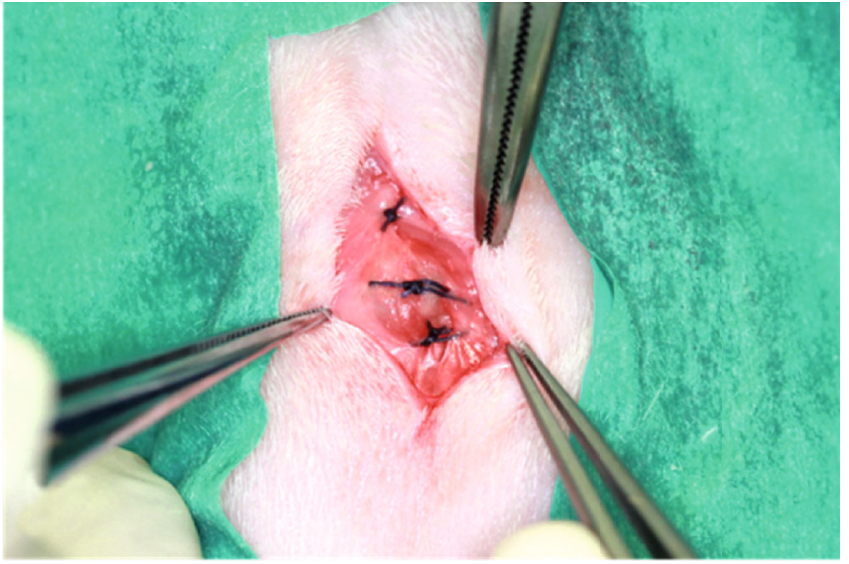

(h)

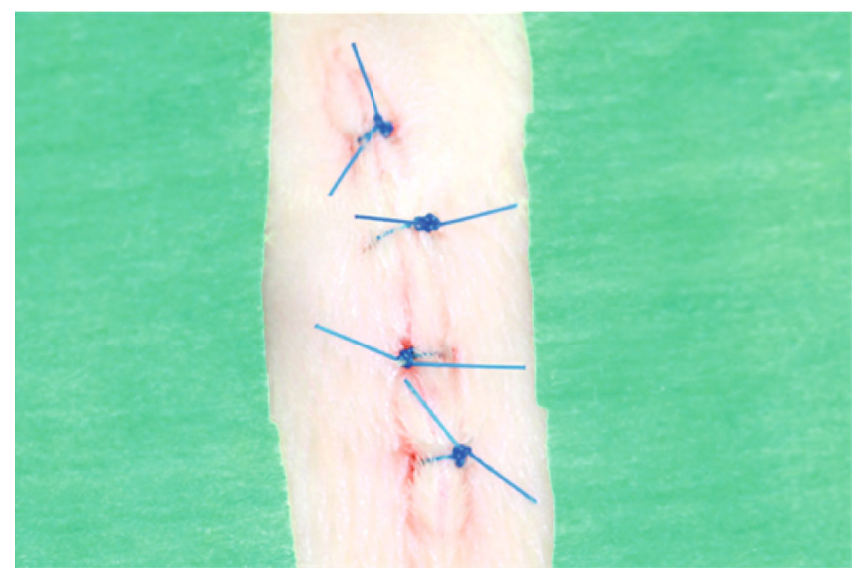

(i)

FIGURE 2: Surgical procedures of dental implant placement. (a) Straight incision on the sagittal crest is performed; (b) the epicranial aponeurosis is carefully dissected from the scalp to use it later for covering the dental implant; (c) after the subperiosteal dissection, the calvaria is exposed; (d) a $3.5 \mathrm{~mm}$ craniotomy is created with a trephine burr and a round burr leaving the inner table intact. Through this hole, the underlying dura is detached from the inner table using a small applicator and a Freer periosteal elevator; (e) a cylinder titanium implant (Straumann ${ }^{\circledR}$ SLA, $6 \mathrm{~mm}$, Basel, Switzerland) is inserted, leaving $2 \mathrm{~mm}$ of the implant extracranially (f); (g) the extracranial part of the implant was covered with a resorbable membrane (Geistlich Bio-Gide ${ }^{\circledR}, 25 \mathrm{~mm} \times 25 \mathrm{~mm}$, Wolhusen, Switzerland); (h) the epicranial aponeurosis was closed over the membrane with single interrupted sutures (Ethilon ${ }^{\circledR}$ ); (i) the wound was closed with single interrupted sutures.

manner, and each specimen was able to make 2 optimal sections.

2.6. Fluorochrome Labeling Analysis. Fluorochrome labeling analysis was performed to analyze bone growth rate and the dynamic bone formation using the Zeiss LSM 710 Upright Confocal Microscope and the Zeiss LSM 780 Inverted Confocal Microscope (Zeiss, Oberkochen, Germany). Images analysis was performed using the ZEN 2012 software. Bone growth rates were calculated by dividing the measured average distance between two sequenced fluorochrome labeling lines by the interval days between two injections [13].

2.7. Histological Examinations. After laser confocal imaging, the sections were stained with $1 \%$ toluidine blue in $60^{\circ} \mathrm{C}$ water bath for 40 minutes and mounted with Permount ${ }^{\circledR}$ after complete air-dry overnight $\left(37^{\circ} \mathrm{C}\right)$. Microscope images were taken using the Nikon Eclipse LV100 POL (Nikon, Tokyo, Japan).

Osseointegration assessment was conducted by measuring the bone-to-implant contact (BIC), which was calculated as the percentages of mineralized bone in direct contact with the implant surface. Images were analyzed using ImageJ image analysis system (ImageJ 1.33u; National Institutes of Health, Bethesda, MD) to measure the BIC quantitatively according to the protocol described in the previous studies [17-19]. BIC on both sides of the dental implant in both cortical and cancellous calvarial bone [20] was measured and subjected to statistical analysis.

2.8. Statistical Analysis. The IBM SPSS statistics software (version 24.0, IBM Crop, Armonk: NY, USA) was used to perform the statistical analysis. Comparison of BMD, microstructure, bone growth rates, and incidence of osteonecrosis between groups was performed by independent $t$-test at a 

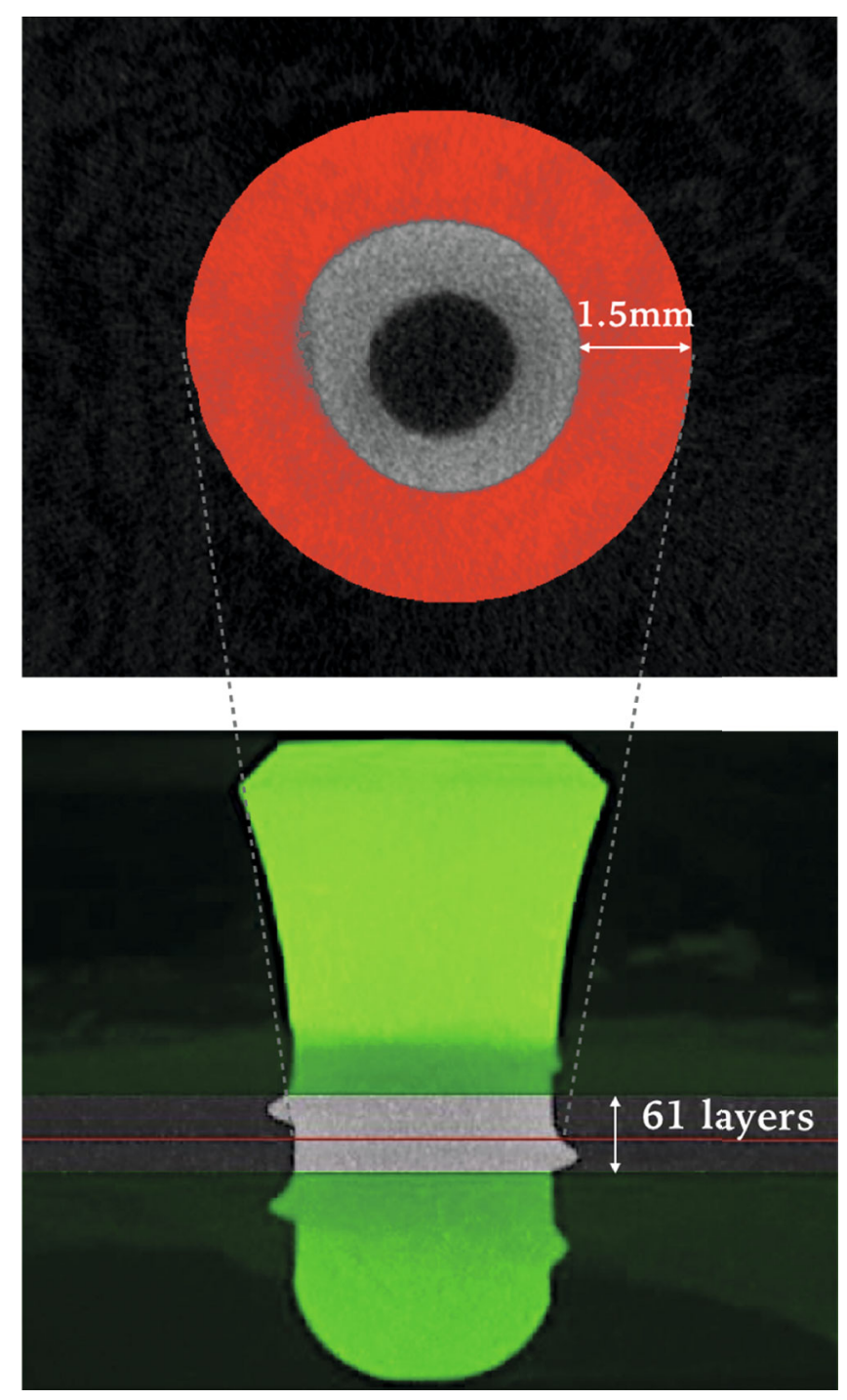

FIgURE 3: The region of interest (ROI) is selected from the surface of the dental implant to the distance of $1.5 \mathrm{~mm}$ away from the implant ((a) red area, cross section view) centered on the midline of the trabecular area on the horizontal level ((b) red line, frontal view). In total, 61 layers are selected.

significance level of 0.05 . The analysis was reviewed by an independent statistician.

\section{Results}

3.1. Clinical Observations. All animals recovered well and went through the whole experiment uneventfully except for one in the ZA long-term group was found dead on the next day following surgery. All surgical sites healed well. No signs of infection, inflammation, or dehiscence were observed. Sutures were removed uneventfully two weeks postoperation.

3.2. Micro-CT Assessment. The 3D micro-CT images were reconstructed from region of interest (ROI) (Figure 4). The results of BMD and other bone microstructure indices repre- senting implant osseointegration were analyzed using independent sample $t$-test (version 24.0, IBM Crop, Armonk: NY, USA). The results were summarized in Table 2. The intergroup comparison showed that in the ZA long-term subgroup, BV/TV, BMD, Tb.N, and Tb.Sp values were higher than that in the control long-term subgroup; however, the differences were not statistically significant (Figure 5). Similarly, no significant differences were found in comparison between the ZA short-term group and the control shortterm group.

3.3. Fluorochrome Labeling Analysis. The distances between two sequenced fluorochrome labeling were measured. The average bone growth rates were calculated by dividing the amount of days in between the two sequenced fluorochrome labeling solution injections (Figure 6). In general, ZAtreated group (both at week 4 and week 8) demonstrated significant reduced bone growth rates compared to control counterparts in all time intervals (weeks 1-2, weeks 2-3, weeks 2-4, and weeks 4-6) (Table 3, Figure 6). In the intragroup comparison, the averaged bone growth rates in each subgroup were used. Significant lower bone growth rates in the ZA short-term subgroup (from week 1 to week 3) were seen compared to ZA long-term subgroup (from week 2 to week 6), while no significant difference was detected between control long-term and control shortterm subgroups (Figure 7).

3.4. Histomorphological Analysis. Histological images demonstrated comparable mineralization along the dental implant surface on both sides in both groups (Figure 8). The results of BIC were measured using the 10x objective of the microscope and are summarized in Table 4. Statistical analysis is shown in Figure 9. Significant lower BIC was found in ZA-treated animals compared to controlled animals at week 4, but then it increased to a level that was slightly lower than their control counterparts at week 8 , and the difference at the later time point was found not significant at statistic level. When results were compared to intragroup, no significant difference was detected in neither the control group nor the ZA group.

\section{Discussion}

The understanding of the effect of high-dose BPs on osteointegration of dental implant is very limited. Clinicians desire to have more evidence on whether the success rates of dental implant therapy are affected in patient receiving high-dose BP treatment.

BPs were found to decrease bone turnover rates and thus increase bone mineral density significantly in osteoporotic patients in the first year of treatment and then reaches a plateau [21-22]. Similar findings were reported in animal studies which showed increased BMD, BV/TV, and Tb.Th, in osteoporotic animals (induced by ovariectomy surgery) treated with BPs [23-25]. Our previous studies showed significantly increased bone mineral density in ovariectomized animals treated with BPs [26-28]. In another animal study [29] using a tumor model, significant increase in BMD, 
Control
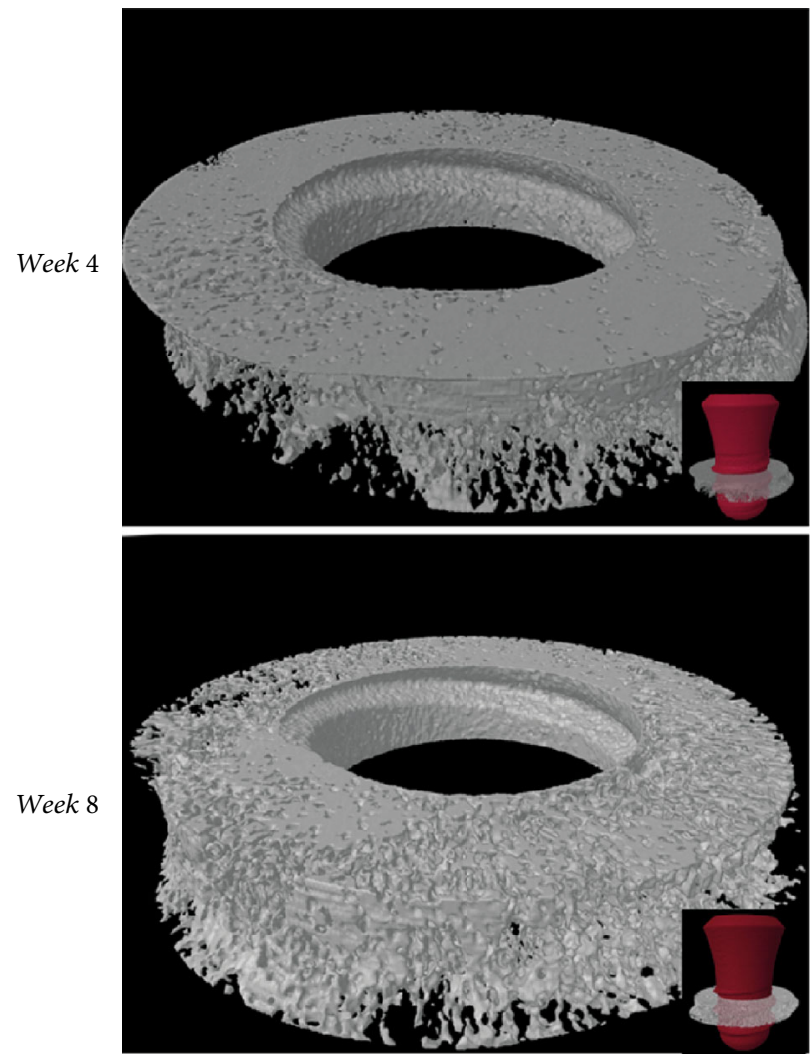

FIGURE 4: The 3D model of each specimen is generated from the region of interest (ROI) defined in this study.
ZA-treated
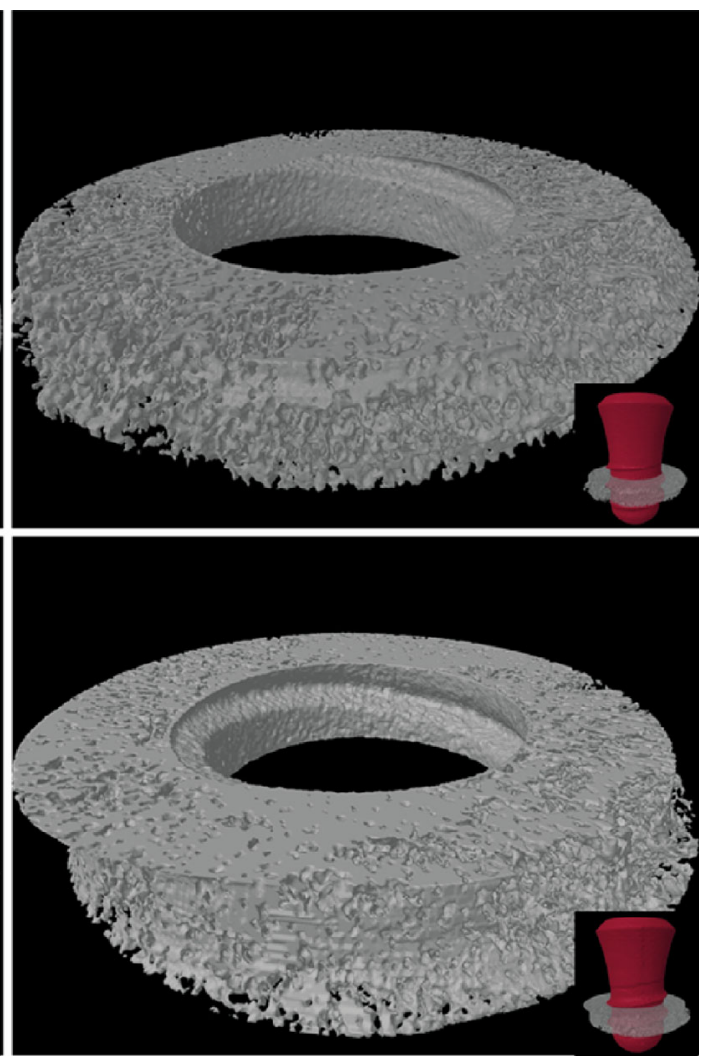

TABLE 2: Statistical analysis result of bone mineral density (BMD) at the calvarial site and other bone microstructure indices (mean \pm SD).

\begin{tabular}{lccccc}
\hline $\begin{array}{l}\text { Groups } \\
\text { Parameters }\end{array}$ & Control short-term & Control long-term & ZA short-term & ZA long-term & $P$ value* \\
\hline BMD & $0.63 \pm 0.08$ & $0.61 \pm 0.07$ & $0.66 \pm 0.05$ & $0.63 \pm 0.02$ & 0.593 \\
BV/TV & $0.56 \pm 0.07$ & $0.50 \pm 0.07$ & $0.55 \pm 0.06$ & $0.52 \pm 0.03$ & 0.776 \\
Tb.Th (mm) & $0.112 \pm 0.012$ & $0.106 \pm 0.009$ & $0.105 \pm 0.002$ & $0.106 \pm 0.004$ & 0.215 \\
Tb.N (1/mm) & $5.00 \pm 0.42$ & $4.71 \pm 0.30$ & $5.25 \pm 0.64$ & $4.93 \pm 0.42$ & 0.490 \\
Tb.Sp (mm) & $0.11 \pm 0.02$ & $0.12 \pm 0.02$ & $0.12 \pm 0.03$ & $0.13 \pm 0.02$ & 0.425 \\
\hline
\end{tabular}

${ }^{*} P$ value between control group and ZA group at week $4 .{ }^{* *} P$ value between control group and ZA group at week 8. BMD: bone mineral density; BV/TV: bone volume/tissue volume, bone volume fraction; Tb.Th: trabecular thickness; Tb.N: trabecular number; Tb.Sp: trabecular separation.

BV/TV, Tb.Th, and Tb.N were detected in tumor-bearing animals treated with BPs, whereas in the control healthy animals, BP treatment did not significantly change the bone mineral density and other trabecular architecture.

In the present study, animals in the long-term ZA group demonstrated increase in BV/TV, Tb.Th, and BMD, but not statistically significant when compared with that in the control group. In the short-term ZA group, BV/TV, BMD, and Tb.N showed slight increase as well; however, Tb.Th declined slightly in comparison with that in the control group. This result may be due to the nonsignificant effect of BPs on normal bone tissue in accordance with the previ- ous mentioned findings. Secondly, these parameters relate closely to the trabecular bones, which are comparatively much less in calvarial bones than in long bones, therefore makes the calculated results less variant. However, measures have been done to minimize the interference of cortical bones: ROI was selected centered on the midline of the trabecular zone horizontally. Another reason would be the relatively small sample size of this study. Lastly, the artifact caused by the metal implant may also affect the accuracy of the results.

Bone growth rates of the calvarial bone dropped significantly when animals were treated with BPs both long- 

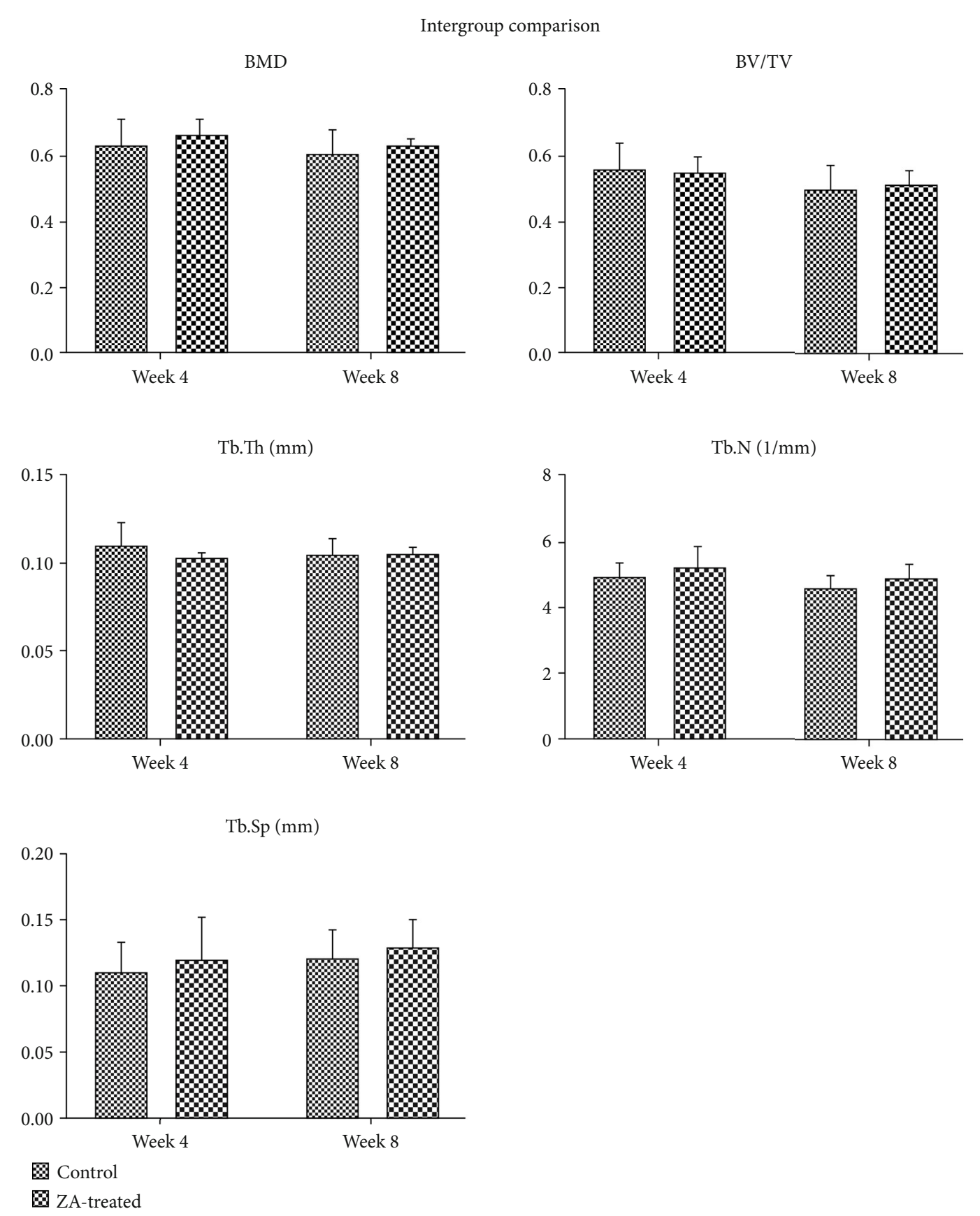

FIGURE 5: Intergroup comparison of BMD, BV/TV, Tb.Th, Tb.N, and Tb.Sp in the calvarial bone between control and ZA-treated groups at the time point of week 4 and week 8 . No significant difference is detected in all the assessed parameters.

termly and short-termly in the present study. The reduced bone growth rates mean reduced new bone formation, which is in conformity with the findings in other studies concerning the mechanism of bisphosphonate actions [30-33]. The decreased bone formation caused by BPs may be explained why BV/TV and BMD showed no significant increase even when high doses of BPs were given in our study. However, the reduced bone formation does not seem to relate to the diminished osteoblastic activity, which was suspected to be caused by the application of
BPs. Actually, many studies described an improved proliferation of osteoblasts $[1,34,35]$ and an inhibitory effect on apoptosis of osteocytes and osteoblasts [36, 37]. Therefore, this bone formation reduction was thought to respond to the declined bone resorption and the following diminished bone remodeling.

Initially at week 4 , BIC was found significantly lower in the ZA-treated animals compared to controlled animals; it may reveal an adverse effect of ZA on osseointegration in the short term. However, BIC demonstrated a gain from 
(a)

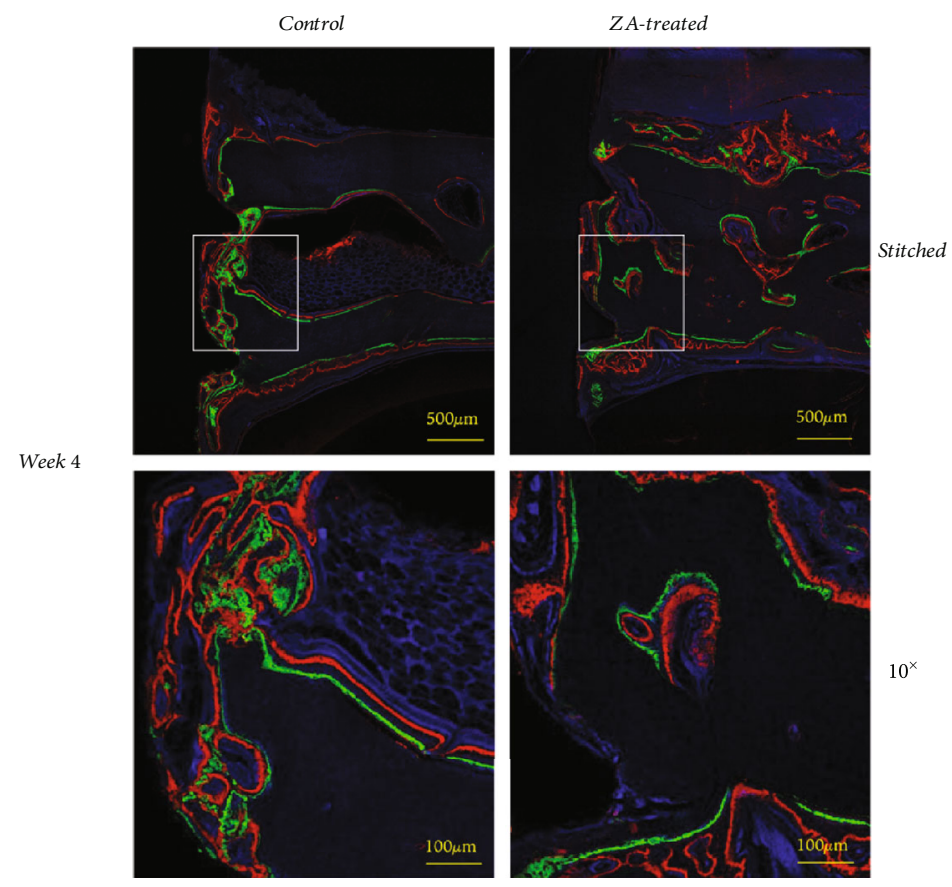

(a)

(b)

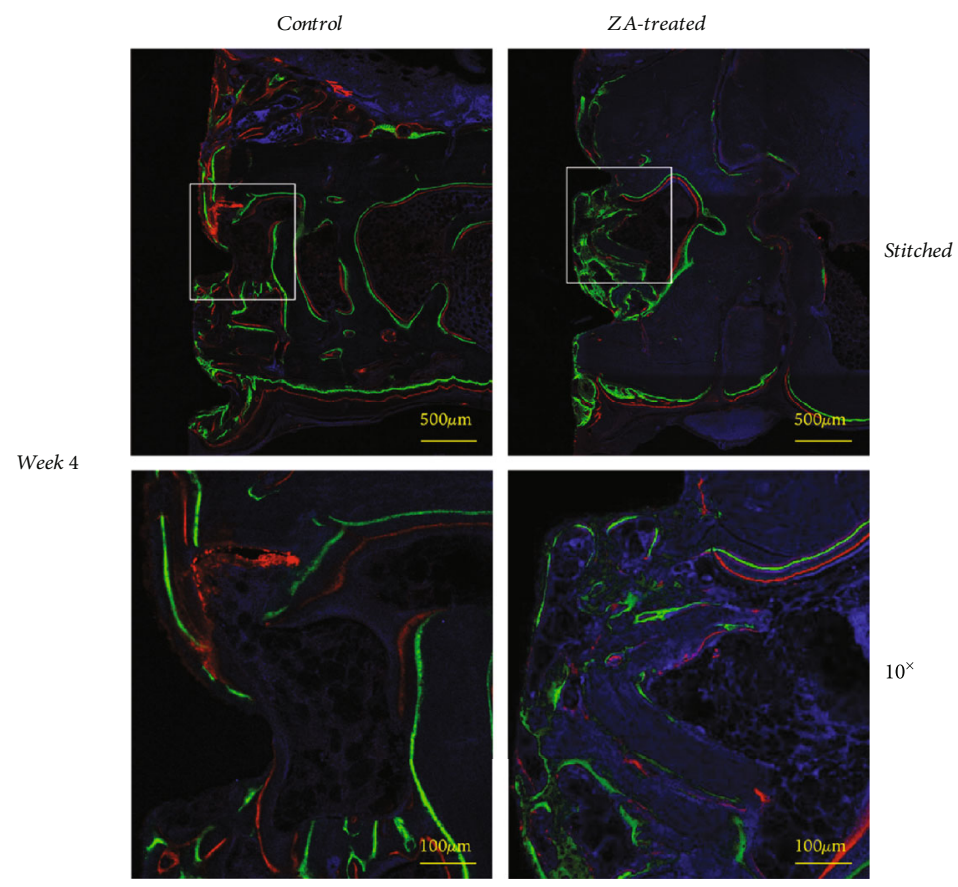

(b)

FIGURE 6: (a) Fluorochrome labeling analysis at week 4 is performed using 10x objective of Zeiss LSM 710 Upright Confocal Microscope, and upper images are stitched after using the tile scan function of the Zeiss LSM 780 Inverted Confocal Microscope. (b) Fluorochrome labeling analysis at week 8 is performed using 10x objective of Zeiss LSM 710 Upright Confocal Microscope, and upper images are stitched after using the tile scan function of the Zeiss LSM 780 Inverted Confocal Microscope. 
TABLE 3: Bone growth rates measured by fluorochrome labeling analysis (mean $\pm \mathrm{SD})$.

\begin{tabular}{lcccc}
\hline \multirow{2}{*}{ Group statistics } & \multicolumn{3}{c}{ Bone growth rates $(\mu \mathrm{m} /$ day $)$} & $t$-test \\
& Groups & $N$ & Mean $\pm S D$ & Sig. (2-tailed)* \\
\hline \multirow{2}{*}{ Weeks 1-2 } & CS & 5 & $2.68 \pm 0.25$ & 0.000 \\
& ZS & 5 & $1.10 \pm 0.03$ & \\
Weeks 2-3 & CS & 5 & $1.85 \pm 0.17$ & 0.000 \\
Weeks 2-4 & ZS & 5 & $0.86 \pm 0.08$ & \\
& CL & 5 & $2.45 \pm 0.22$ & 0.000 \\
Weeks 4-6 & ZL & 4 & $1.77 \pm 0.11$ & \\
& CL & 5 & $1.85 \pm 0.23$ & 0.000
\end{tabular}

CS: control short-term group; ZS: ZA short-term group; CL: control longterm group; ZL: ZA long-term group. ${ }^{*} P$ value.

week 4 to week 8 (though not statistically significant), to a level that was similar though slightly lower than the control counterparts; in the meantime, BIC decreased in the control group from week 4 to week 8 . The gain of BIC in ZA group may be related to the significantly higher bone growth rates in the $\mathrm{ZA}$ long-term group compared to ZA short-term group. At week 8, BIC in the ZA group showed no significant difference in comparison with the control group. This result suggests that ZA may have an unfavorable effect on osseointegration in the short term. However, this effect seemed to diminish in the long term. How the BIC will change in the longer-term needs further investigations.

Currently, a history of oral or intravenous BP use is not considered an absolute contraindication for dental implant insertion. Osseointegration of dental implants was not significantly affected in these patients according to a systematic review [38]. However, current available studies are of levels III to II, which are not strong enough to support any conclusions.

Ayan et al. [39] reported a significant increase of BIC in tibia at week 2 and week 4 after a single dose of systemic ZA in rabbit model. In another animal study [40], implants were placed in the tibia of ovariectomized rabbit, and systemic ZA (single-dose infusion) was demonstrated to improve osseointegration as well. Similar results were reported in other studies [40-42]. These animal studies varied in BP type, dosage, and route of administration, and the implants were placed in the tibia, which exhibited a lower remodeling rate than that in jaw bones.

Different results were observed in animal studies that placed implants in jaw bones. Using an ovariectomized rat model with implant placement in mandibles, Cardemil et al. [43] demonstrated a significantly lower BIC in the ZA group (single IV dose) compared to control group at 14 days. The BIC showed some growth to a level higher than the control counterparts at 28 days and exhibited no significant difference at this time point. The results in our study were similar except that the reduction in BIC was not significant in the ZA group at the early time point
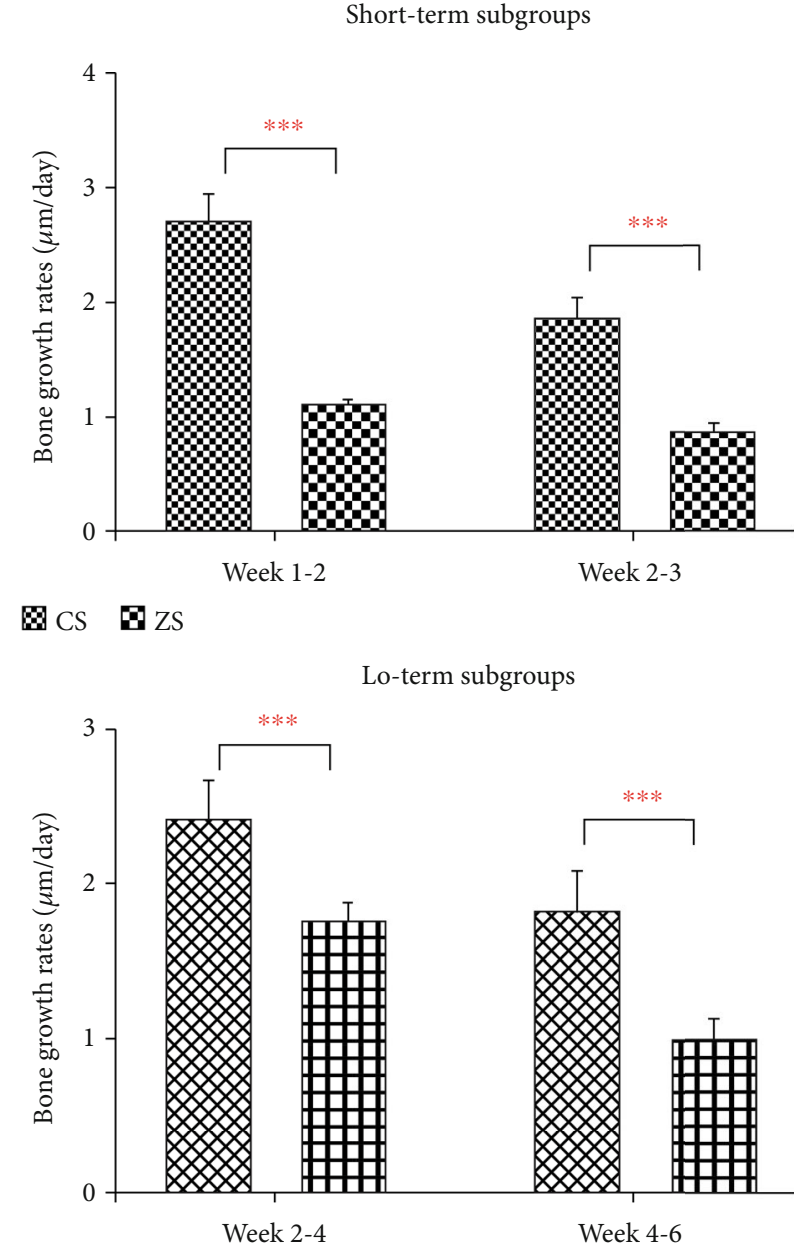

$\otimes C L \quad \boxplus Z L$

Intragroup comparison

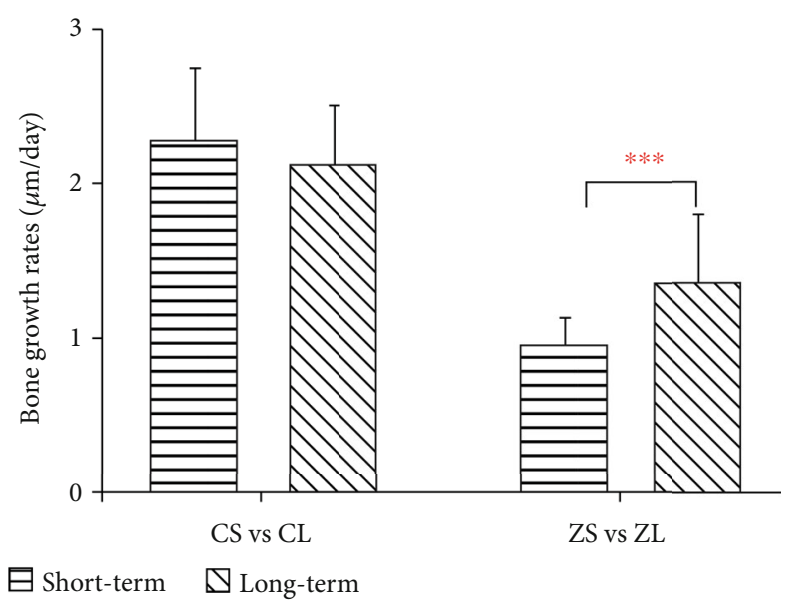

FIGURE 7: Intergroup comparison and intragroup comparison of bone growth rates $(\mu \mathrm{m} /$ day $)$ of the calvarial bone. CS: control short-term subgroup; ZS: ZA short-term subgroup; CL: control long-term subgroup; ZL: ZA long-term subgroup. ${ }^{* * *} P<0.001$.

though the dosage of ZA was much higher in our study. In another study placing implants in the parietal bone of rabbit [44], it was concluded that systemic use of 


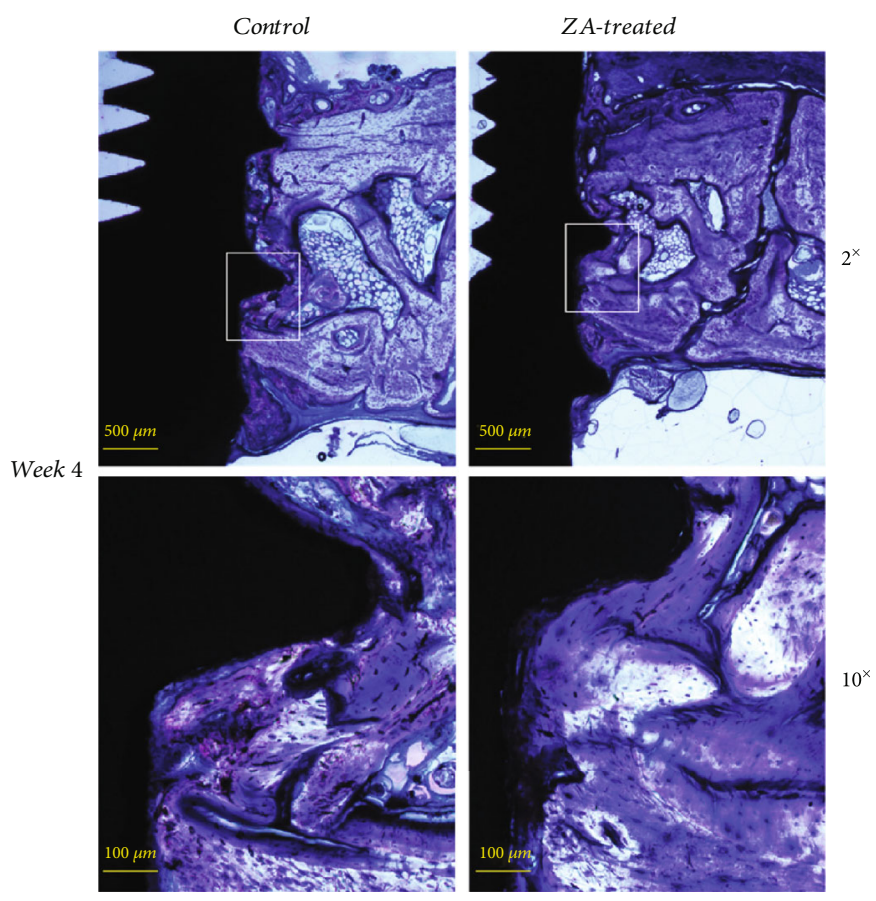

(a)
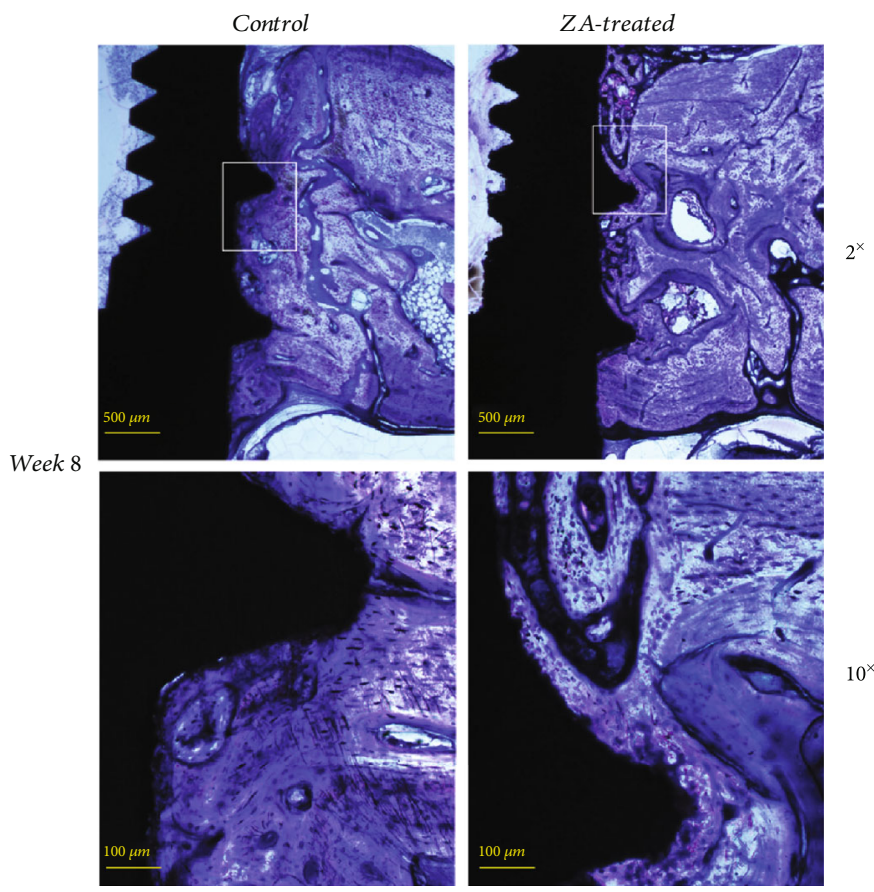

(b)

Figure 8: Histological assessment for the control and ZA-treated groups at week 4 is illustrated in the picture (2x objective), and measurement of bone-to-implant contact (BIC) is made using the 10x objective on both sides of the implant. Histological assessment for the control and ZA-treated groups at week 8 is illustrated as above (2x objective), and measurement of bone-to-implant contact (BIC) is made using the 10x objective on both sides of the implant. Comparable mineralization is shown in both groups and at different time points. 
TABLE 4: Bone-to-implant contact (BIC) in calvarial bone at week 4 and week 8 .

\begin{tabular}{lccccccc}
\hline \multirow{2}{*}{ Group } & \multicolumn{3}{c}{ Week 4 } & \multicolumn{2}{c}{ Week 8 } & \multirow{2}{*}{$P$ value* } \\
& $N$ & Mean & SD & $N$ & Mean & SD & \\
\hline Control & 5 & 0.38 & 0.035 & 5 & 0.35 & 0.063 & 0.401 \\
ZA-treated & 5 & 0.28 & 0.083 & 4 & 0.34 & 0.019 & 0.151 \\
$P$ value** & \multicolumn{4}{c}{0.030} & & 0.815 & \\
\end{tabular}

${ }^{*}$ Intragroup comparison between two time points. ${ }^{* *}$ Intergroup comparison between control and ZA-treated groups.
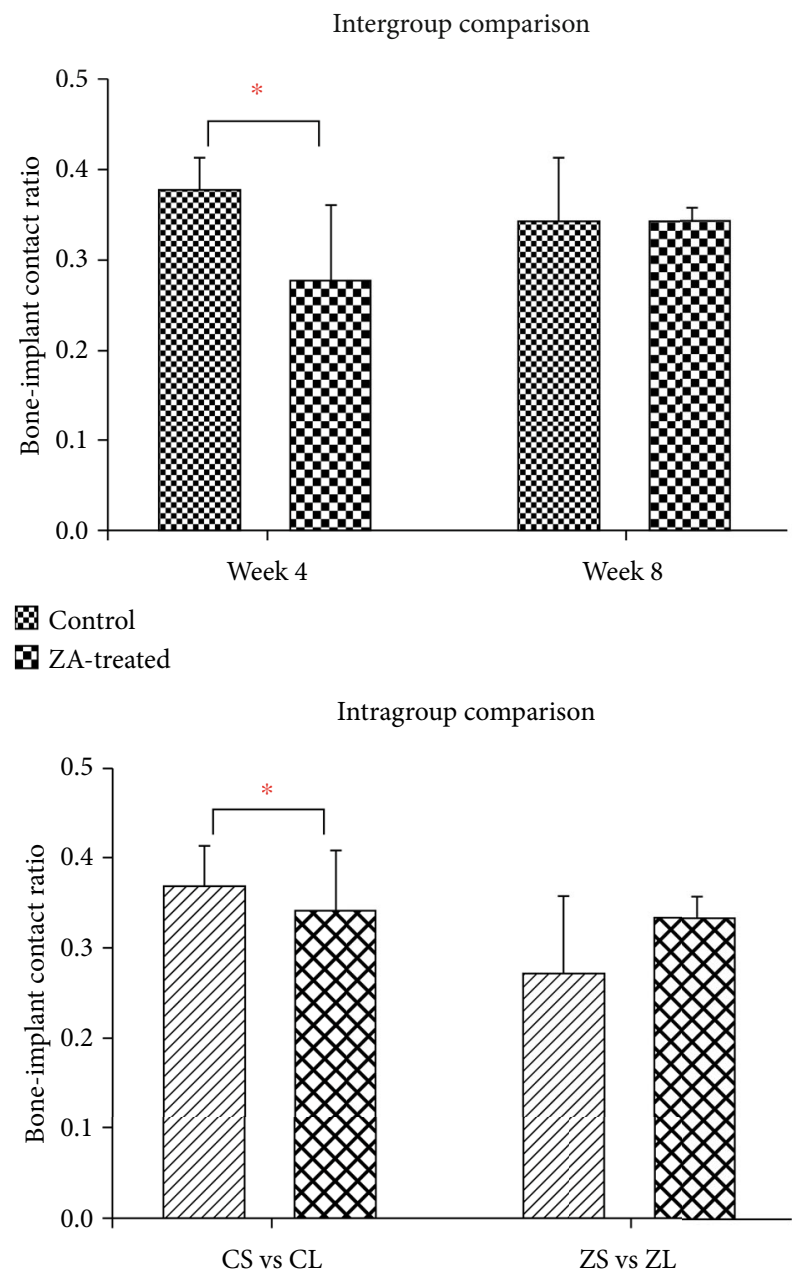

Week 4

FiguRE 9: Intergroup and intragroup comparison of BIC in the calvarial bone. Significant difference is detected in the comparison between control group and ZA group at week 4 but not at week 8 . In the intragroup comparison, no significant difference is found. * $P<0.05$.

alendronate may delay the osseointegration of the newly formed bone.

The results of the BIC may be interpreted in a way that BPs may have an adverse effect on osseointegration in calvarial bone in the short term but this effect tends to diminish or possibly become a positive effect in the long term. However, this interpretation has to be considered with care due to the limited number of animals included in each group. In addition, rabbits exhibit a different composition of bone and a much higher bone turnover rate than human, and translation of the results of rabbits to human subjects needs critical assessment.

In conclusion, high-dose ZA treatment inhibits bone growth rates and showed an adverse effect on osseointegration of dental implant in short term. However, no significant effect on bone mineral density or trabecular microarchitecture is detected in the rabbit model with implant placement on the calvarial bone.

\section{Abbreviations}

ZA: $\quad$ Zoledronic acid

Micro-CT: Microcomputed tomography

MRONJ: Medication-related osteonecrosis of the jaws

BP: $\quad$ Bisphosphonate

BMD: $\quad$ Bone mineral density

BV: $\quad$ Bone volume

TV: $\quad$ Tissue volume

BV/TV: Bone volume fraction

BV/TV: Bone volume/tissue volume [14]

Tb.Th: Trabecular thickness

Tb.N: Trabecular number

Tb.Sp: Trabecular separation

ROI: $\quad$ Region of interest

BIC: $\quad$ Bone-to-implant contact.

\section{Data Availability}

The data used to support the findings of this study are included within the article.

\section{Ethical Approval}

This article does not contain any studies with human participants performed by any of the authors. All applicable international, national, and/or institutional guidelines for the care and use of animals are followed. This study was reviewed and approved by the Committee on Use Live Animal for Teaching and Research, the University of Hong Kong (CULATR No. 3774-15).

\section{Consent}

For this type of study, formal consent is not required.

\section{Disclosure}

The study has been presented as an abstract in 96th General Session \& Exhibition of the IADR PAN European Regional Congress. The abstract was presented in HKU Postgraduate Thesis.

\section{Conflicts of Interest}

The authors declare that there is no conflict of interest regarding the publication of this paper. 


\section{Acknowledgments}

The work is supported by the Seed Fund for Basic Research (201611159015), the University of Hong Kong. The methodology in this study was reviewed by an independent statistician, Samantha Kar Yan Li (Faculty of Dentistry, The University of Hong Kong). The authors would like to acknowledge her for her assistance in the statistical analysis.

\section{References}

[1] G.-I. Im, S. A. Qureshi, J. Kenney, H. E. Rubash, and A. S. Shanbhag, "Osteoblast proliferation and maturation by bisphosphonates," Biomaterials, vol. 25, no. 18, pp. 41054115, 2004.

[2] G. A. Rodan and H. A. Fleisch, "Bisphosphonates: mechanisms of action," The Journal of Clinical Investigation, vol. 97, no. 12, pp. 2692-2696, 1996.

[3] J. E. Fisher, M. J. Rogers, J. M. Halasy et al., "Alendronate mechanism of action: geranylgeraniol, an intermediate in the mevalonate pathway, prevents inhibition of osteoclast formation, bone resorption, and kinase activation in vitro," Proceedings of the National Academy of Sciences, vol. 96, no. 1, pp. 133-138, 1999.

[4] F. P. Coxon, M. H. Helfrich, R. Van't Hof et al., "Protein geranylgeranylation is required for osteoclast formation, function, and survival: inhibition by bisphosphonates and GGTI-298," Journal of Bone and Mineral Research, vol. 15, no. 8, pp. 1467-1476, 2000.

[5] D. Hughes, B. MacDonald, R. Russell, and M. Gowen, "Inhibition of osteoclast-like cell formation by bisphosphonates in long-term cultures of human bone marrow," The Journal of Clinical Investigation, vol. 83, no. 6, pp. 1930-1935, 1989.

[6] A. Schmidt, S. J. Rutledge, N. Endo et al., "Protein-tyrosine phosphatase activity regulates osteoclast formation and function: inhibition by alendronate," Proceedings of the National Academy of Sciences, vol. 93, no. 7, pp. 3068-3073, 1996.

[7] H. B. Kwak, J. Y. Kim, K. J. Kim et al., "Risedronate directly inhibits osteoclast differentiation and inflammatory bone loss," Biological and Pharmaceutical Bulletin, vol. 32, no. 7, pp. 1193-1198, 2009.

[8] H. Fleisch, Bisphosphonates in Bone Disease: From the Laboratory to the Patient, Academic Press, 2000.

[9] P. Papaspyridakos, C.-J. Chen, M. Singh, H.-P. Weber, and G. Gallucci, "Success criteria in implant dentistry: a systematic review," Journal of Dental Research, vol. 91, no. 3, pp. 242-248, 2011.

[10] P.-I. Brånemark, U. Breine, R. Adell, B. Hansson, J. Lindström, and $\AA$. Ohlsson, "Intra-osseous anchorage of dental prostheses: I. Experimental studies," Scandinavian Journal of Plastic and Reconstructive Surgery, vol. 3, no. 2, pp. 81-100, 2009.

[11] A. Stavropoulos, K. Bertl, P. Pietschmann, N. Pandis, M. Schiødt, and B. Klinge, "The effect of antiresorptive drugs on implant therapy: systematic review and meta-analysis," Clinical Oral Implants Research, vol. 29, no. S18, pp. 54-92, 2018.

[12] S. M. van Gaalen, M. C. Kruyt, R. E. Geuze, J. D. de Bruijn, J. Alblas, and W. J. Dhert, "Use of fluorochrome labels in in vivo bone tissue engineering research," Tissue engineering Part B: Reviews, vol. 16, no. 2, pp. 209-217, 2010.
[13] J. Y. Li, E. H. N. Pow, L. W. Zheng, L. Ma, D. L. W. Kwong, and L. K. Cheung, "Dose-dependent effect of radiation on titanium implants: a quantitative study in rabbits," Clinical Oral Implants Research, vol. 25, no. 2, pp. 260-265, 2014.

[14] A. M. Parfitt, M. K. Drezner, F. H. Glorieux et al., "Bone histomorphometry: standardization of nomenclature, symbols, and units: report of the ASBMR Histomorphometry Nomenclature Committee," Journal of Bone and Mineral Research, vol. 2, no. 6, pp. 595-610, 1987.

[15] T. Hildebrand and P. Rüegsegger, "A new method for the model-independent assessment of thickness in threedimensional images," Journal of Microscopy, vol. 185, no. 1, pp. 67-75, 2003.

[16] A. Parfitt, C. Mathews, A. Villanueva, M. Kleerekoper, B. Frame, and D. Rao, "Relationships between surface, volume, and thickness of iliac trabecular bone in aging and in osteoporosis. Implications for the microanatomic and cellular mechanisms of bone loss," The Journal of Clinical Investigation, vol. 72, no. 4, pp. 1396-1409, 1983.

[17] P. M. Duarte, J. B. C. Neto, P. F. Gonçalves, E. A. Sallum, and F. H. Nociti Jr., "Estrogen deficiency affects bone healing around titanium implants: a histometric study in rats," Implant Dentistry, vol. 12, no. 4, pp. 340-346, 2003.

[18] P. M. Duarte, P. F. Gonçalves, M. Zaffalon Casati, E. A. Sallum, and F. H. Nociti Jr., "Age-related and surgically induced estrogen deficiencies may differently affect bone around titanium implants in rats," Journal of Periodontology, vol. 76, no. 9, pp. 1496-1501, 2005.

[19] J. B. César-Neto, B. B. Benatti, E. A. Sallum, A. W. Sallum, and F. H. Nociti Jr., "Bone filling around titanium implants may benefit from smoking cessation: a histologic study in rats," Journal of Periodontology, vol. 76, no. 9, pp. 1476-1481, 2005.

[20] F. V. Ribeiro, J. B. César-Neto, F. H. Nociti et al., "Selective cyclooxygenase-2 inhibitor may impair bone healing around titanium implants in rats," Journal of Periodontology, vol. 77, no. 10, pp. 1731-1735, 2006.

[21] M. R. McClung, "Current bone mineral density data on bisphosphonates in postmenopausal osteoporosis," Bone, vol. 19, no. 5, Supplement 1, pp. 195S-198S, 1996.

[22] R. P. Tonino, P. J. Meunier, R. Emkey et al., "Skeletal benefits of alendronate: 7-year treatment of postmenopausal osteoporotic women," The Journal of Clinical Endocrinology \& Metabolism, vol. 85, no. 9, pp. 3109-3115, 2000.

[23] E. Perilli, V. Le, B. Ma, P. Salmon, K. Reynolds, and N. L. Fazzalari, "Detecting early bone changes using in vivo micro-CT in ovariectomized, zoledronic acid-treated, and shamoperated rats," Osteoporosis Int, vol. 21, no. 8, pp. 13711382, 2010.

[24] S. Hornby, G. Evans, S. Hornby, A. Pataki, M. Glatt, and J. Green, "Long-term zoledronic acid treatment increases bone structure and mechanical strength of long bones of ovariectomized adult rats," Calcified Tissue International, vol. 72, no. 4, pp. 519-527, 2003.

[25] J. Brouwers, F. Lambers, J. A. Gasser, B. Van Rietbergen, and R. Huiskes, "Bone degeneration and recovery after early and late bisphosphonate treatment of ovariectomized wistar rats assessed by in vivo micro-computed tomography," Calcified Tissue International, vol. 82, no. 3, pp. 202-211, 2008.

[26] C. L. Li, W. W. Lu, C. J. Seneviratne, W. K. Leung, R. A. Zwahlen, and L. W. Zheng, "Role of periodontal disease in bisphosphonate-related osteonecrosis of the jaws in 
ovariectomized rats," Clinical Oral Implants Research, vol. 27, no. 1, pp. 1-6, 2016.

[27] R. Nianjing, Role of Periapical Diseases in BisphosphonatesRelated Osteonecrosis of the Jaws, The University of Hong Kong, 2017.

[28] L. Huo, Effect of bisphosphonates on periodontitis in a compromised rat model, The University of Hong Kong, Pokfulam, Hong Kong, 2016.

[29] E. Won, L. Wise-Milestone, M. K. Akens et al., "Beyond bisphosphonates: photodynamic therapy structurally augments metastatically involved vertebrae and destroys tumor tissue," Breast Cancer Research and Treatment, vol. 124, no. 1, pp. 111-119, 2010.

[30] T. Storm, T. Steiniche, G. Thamsborg, and F. Melsen, "Changes in bone histomorphometry after long-term treatment with intermittent, cyclic etidronate for postmenopausal osteoporosis," Journal of Bone and Mineral Research, vol. 8, no. 2, pp. 199-208, 1993.

[31] R. Boyce, C. Paddock, J. Gleason, W. Sletsema, and E. Eriksen, "The effects of risedronate on canine cancellous bone remodeling: three-dimensional kinetic reconstruction of the remodeling site," Journal of Bone and Mineral Research, vol. 10, no. 2, pp. 211-221, 1995.

[32] S. M. Ott, "Clinical effects of bisphosphonates in involutional osteoporosis," Journal of Bone and Mineral Research, vol. 8, no. S2, pp. S597-S606, 1993.

[33] R. Balena, B. C. Toolan, M. Shea et al., "The effects of 2year treatment with the aminobisphosphonate alendronate on bone metabolism, bone histomorphometry, and bone strength in ovariectomized nonhuman primates," The Journal of Clinical Investigation, vol. 92, no. 6, pp. 2577-2586, 1993.

[34] N. Maruotti, A. Corrado, A. Neve, and F. P. Cantatore, "Bisphosphonates: effects on osteoblast," European Journal of Clinical Pharmacology, vol. 68, no. 7, pp. 1013-1018, 2012.

[35] N. Giuliani, M. Pedrazzoni, G. Negri, G. Passeri, M. Impicciatore, and G. Girasole, "Bisphosphonates stimulate formation of osteoblast precursors and mineralized nodules in murine and human bone marrow cultures in vitro and promote early osteoblastogenesis in young and aged mice in vivo," Bone, vol. 22, no. 5, pp. 455-461, 1998.

[36] L. I. Plotkin, R. S. Weinstein, A. M. Parfitt, P. K. Roberson, S. C. Manolagas, and T. Bellido, "Prevention of osteocyte and osteoblast apoptosis by bisphosphonates and calcitonin," The Journal of Clinical Investigation, vol. 104, no. 10, pp. 13631374, 1999.

[37] T. Bellido and L. I. Plotkin, "Novel actions of bisphosphonates in bone: preservation of osteoblast and osteocyte viability," Bone, vol. 49, no. 1, pp. 50-55, 2011.

[38] G. K. Chadha, A. Ahmadieh, S. Kumar, and P. P. Sedghizadeh, "Osseointegration of dental implants and osteonecrosis of the jaw in patients treated with bisphosphonate therapy: a systematic review," Journal of Oral Implantology, vol. 39, no. 4, pp. 510-520, 2013.

[39] M. Ayan, D. Dolanmaz, A. Mihmanll, A. Ayan, and M. Kürkçü, "The effect of systemically administrated zoledronic acid on the osseointegration of dental implants," Oral Diseases, vol. 18, no. 8, pp. 802-808, 2012.

[40] A. Yıldız, E. Esen, M. Kürkçü, İ. Damlar, K. Dağlığlu, and T. Akova, "Effect of zoledronic acid on osseointegration of titanium implants: an experimental study in an ovariectomized rabbit model," Journal of Oral and Maxillofacial Surgery, vol. 68 , no. 3, pp. 515-523, 2010.

[41] J. P. Li, P. Li, J. Hu et al., "Early healing of hydroxyapatitecoated implants in grafted bone of zoledronic acid-treated osteoporotic rabbits," Journal of Periodontology, vol. 85, no. 2, pp. 308-316, 2014.

[42] M. Qi, J. Hu, J. Li et al., "Effect of zoledronate acid treatment on osseointegration and fixation of implants in autologous iliac bone grafts in ovariectomized rabbits," Bone, vol. 50, no. 1, pp. 119-127, 2012.

[43] C. Cardemil, O. M. Omar, B. Norlindh, C. L. Wexell, and P. Thomsen, "The effects of a systemic single dose of zoledronic acid on post-implantation bone remodelling and inflammation in an ovariectomised rat model," Biomaterials, vol. 34, no. 5, pp. 1546-1561, 2013.

[44] N. Mardas, F. Schwarz, A. Petrie, A. R. Hakimi, and N. Donos, "The effect of SLActive surface in guided bone formation in osteoporotic-like conditions," Clinical Oral Implants Research, vol. 22, no. 4, pp. 406-415, 2011. 